\title{
Clinical Profile of Diabetic Nephropathy and Correlation With Intrarenal Resistivity Index by Duplex Ultrasonography
}

\author{
Tushar P Raut ${ }^{\mathrm{a}}$, Tushar B Patil ${ }^{\mathrm{a}, \mathrm{c}}$, Rajashree S Khot ${ }^{\mathrm{a}}$, Kiran M Sargar ${ }^{\mathrm{b}}$, \\ Mangesh B Patila, Yogendra V Bansod ${ }^{a}$
}

\begin{abstract}
Background: To study the clinical profile of Diabetic nephropathy and the correlation of intrarenal resistivity index with parameters of renal dysfunction like Glomerular filtration rate, Serum Creatinine, micro and macroalbuminuria.

Methods: This was a cross sectional observational study carried out over a period of 2 years. Patients of type 2 Diabetes as per WHO criteria who had nephropathy were included. A Detailed history was taken and clinical examination was done. Urine routine and microscopic examination and biochemical investigations were done. Patients were subjected to ultrasound of kidneys and renal doppler.
\end{abstract}

Results: A total of 160 patients of type 2 diabetes with diabetic nephropathy were studied. They were divided into two groups based on intrarenal resistivity index (IRI) by duplex ultrasonography as Group I: Patients with IRI $\leq 0.70(\mathrm{n}=72)$ and Group II: Patients with IRI $>0.70(n=88)$. Mean age in group I was $50.42 \pm 4.89$ and in group II was $60.34 \pm 7.92(\mathrm{P}=0.000)$. Mean duration of diabetes mellitus in group I was $4.57 \pm 3.65$ years and $11.25 \pm 6.97$ years in group II. Mean systolic BP in group II was $144.09 \pm 16.79 \mathrm{mmHg}$ whereas in group I it was $128.47 \pm 13.07 \mathrm{mmHg}, 80.55 \%$ in group I were in the early stage of nephropathy whereas $69.32 \%$ patients of group II were in established stage of nephropathy. On multivariate analysis, factors which independently affected IRI were age, Hypertension, Complications - Coronary artery disease and retinopathy, Macroalbuminuria, Decreased creatinine clearance.

Conclusions: Intrarenal resistivity index as assessed by duplex ultrasonography is a non-invasive parameter that can be correlated

Manuscript accepted for publication October 2, 2012

${ }^{a}$ Department of Medicine, Government Medical College, Nagpur, Maharashtra, India

${ }^{\mathrm{b}}$ Department of Radiodiagnosis, Government Medical College, Nagpur, Maharashtra, India

${ }^{c}$ Corresponding author: Tushar B. Patil, Plot No. 9, Rashtrasant Nagar, Godhani Road, Zingabai Takli, Nagpur-440030, Maharashtra, India.

Email: dr.tushar42@rediffmail.com

doi: http://dx.doi.org/10.4021/wjnu41w with the clinical profile and biochemical parameters of renal dysfunction type II diabetes mellitus with diabetic nephropathy.

Keywords: Diabetes mellitus; Diabetic nephropathy; Duplex ultrasound; Intrarenal resistivity index

\section{Introduction}

Diabetes has emerged as one of the major health care problems in India. According to the Diabetes atlas published by the International Diabetes Federation (IDF), there are estimated 40.7 million persons with diabetes in India in 2007 and this number is predicted to rise to almost 60.9 million by 2025 . It is also known that almost $50 \%$ of the people with diabetes remain undetected and some may even present with microvascular and macrovascular complications at the time of diagnosis [1]. In the CURES study the prevalence of overt nephropathy was $2.2 \%$ while that of microalbuminuria was $26.9 \%$.

Assuming that 40 million people in India have Diabetes, this translates to 0.8 million with nephropathy. Thus the burden due nephropathy is very high in India due to sheer number of people with diabetes. Diabetic nephropathy is a progressive kidney disease caused by angiopathy of capillaries in the kidney glomeruli, characterised by albuminuria which progresses from normoalbuminuria to microalbuminuria to macroalbuminuria ultimately leading to End stage renal disease.

Intrarenal resistivity index (IRI) is a measure of the hemodynamic changes in the renal arteries. In diabetic nephropathy changes in the compliance of the vessels' valve and the resistance of the vessels' valve affects the resistivity index. Hence, early changes in blood flow are picked up by renal Doppler and they reflect the progression of diabetic nephropathy. A lot more has to be studied about Diabetic nephropathy and role a non invasive diagnostic tool like renal Doppler.

Hence this research has been carried out to study the clinical profile of Diabetic nephropathy and the correlation of intrarenal resistivity index with parameters of renal dys- 


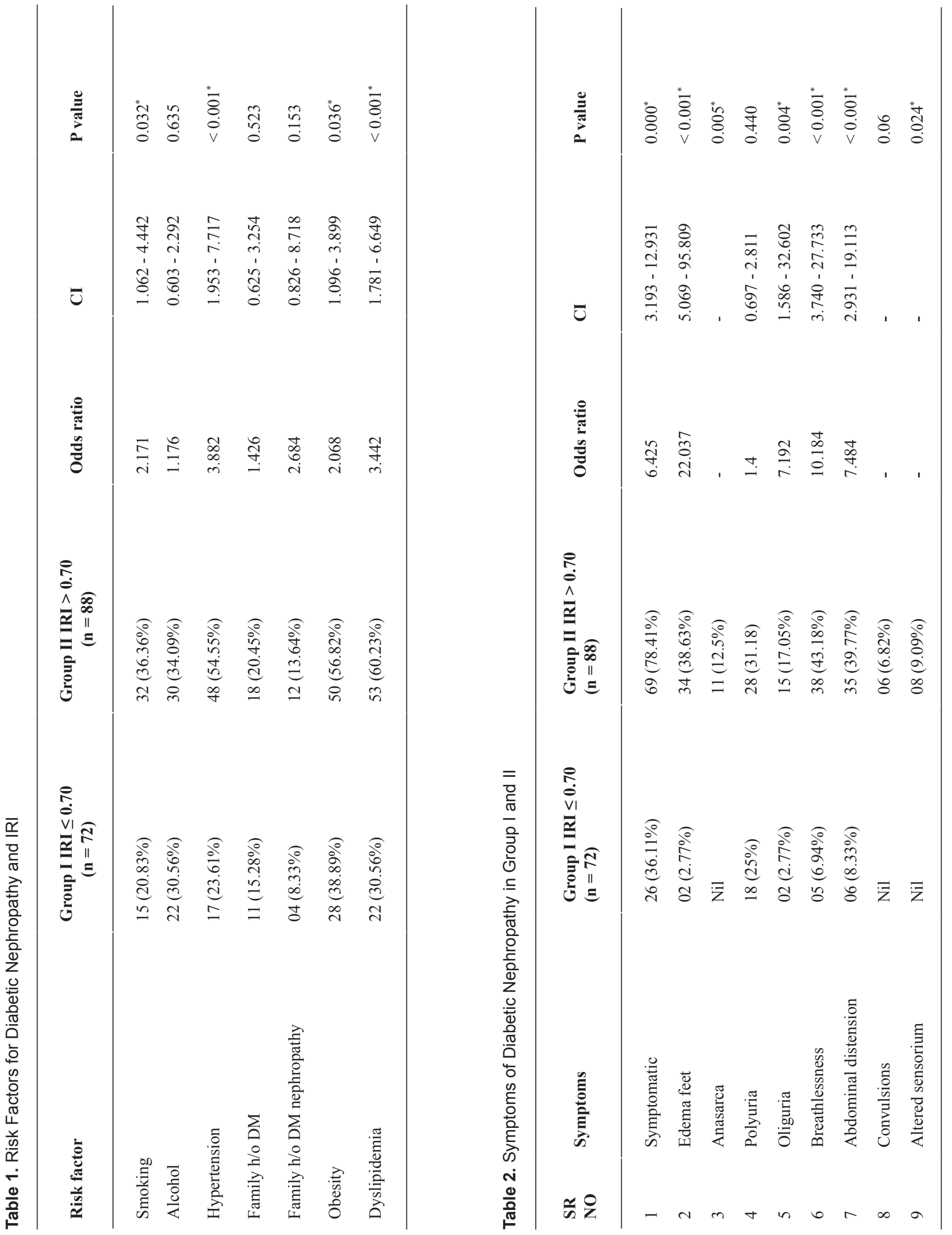


Table 3. Complications of Diabetes

\begin{tabular}{|c|c|c|c|c|c|}
\hline Complication & $\begin{array}{c}\text { Group I IRI } \leq \mathbf{0 . 7 0} \\
\quad(n=72)\end{array}$ & $\begin{array}{c}\text { Group } \text { II IRI }>0.70 \\
(n=88)\end{array}$ & Odds ratio & CI & P value \\
\hline Nil & $50(69.44 \%)$ & $18(20.45 \%)$ & 8.83 & $4.289-18.175$ & $0.000^{*}$ \\
\hline CAD & $01(1.39 \%)$ & $17(19.32 \%)$ & 17.0 & $2.203-131.195$ & $<0.001^{*}$ \\
\hline Retinopathy & $20(27.78 \%)$ & $70(79.54 \%)$ & 10.11 & $4.868-21.001$ & $<0.001^{*}$ \\
\hline Background & 19 & 48 & & & \\
\hline Proliferative & 01 & 22 & & & \\
\hline Neuropathy & $07(9.72 \%)$ & $36(40.90 \%)$ & 6.429 & $2.645-15.623$ & $<0.001^{*}$ \\
\hline PVD & $07(9.72 \%)$ & $16(18.18 \%)$ & 2.063 & $0.799-5.332$ & 0.129 \\
\hline
\end{tabular}

function like Glomerular filtration rate, Serum Creatinine, micro and macroalbuminuria.

\section{Material and Methods}

This was a cross sectional observational study carried out over a period of 2 years. Patients of type 2 Diabetes as per WHO criteria who had nephropathy were included which was defined by: 1) Proteinuria as measured by dipsticks. Urine microalbuminuria in cases of dipstick negative or trace proteinuria. a) Microalbuminuria: 30 - $300 \mathrm{mg} / 24 \mathrm{hrs}$; b) Macroalbuminuria: $>300 \mathrm{mg} / 24 \mathrm{hrs}$. 2) Creatinine clearance as measured by cockcroft-gault formula.

Exclusion criteria: 1) Type 1 diabetes mellitus; 2) Connective tissue disorder like Systemic lupus erythematosus, Systemic sclerosis, rheumatoid arthritis, etc. 3) Renal artery stenosis; 4) Hypertensive nephropathy; 5) Obstructive nephropathy; 6) Drug or toxin induced nephropathy.

A Detailed history regarding symptoms, complications, treatment history and family history was taken and detailed clinical examination was done. Urine routine and microscopic examination was performed and Spot Urine protein analysis by dipsticks was done. Those with dipstick negative urine test were subjected to $24 \mathrm{hrs}$ urine microalbumin assay. Biochemical investigations blood urea, serum creatinine, lipid profile, blood sugar - fasting post meal and ECG were done. The patients were subjected to ultrasound evaluation of kidneys and renal Doppler. Kidney sizes, cortical echotexture and corticomedullary differentiation was described. Colour Doppler was applied on the renal artery and its branches like segmental and arcuate arteries. Spectral wave forms were obtained and PSV, acceleration, delta time was determined. Three different readings for Intrarenal resistivity index were taken and their mean was taken. The mean of IRI of both kidneys gave the final mean IRI.

\section{Statistical analysis}

Patients were divided into 2 groups for comparison, group I with normal IRI and group II with IRI $>0.70$. Data was reported as means $\pm \mathrm{SD}$ when normally distributed. For categorical data, chi-square test was used and Fischer exact test for small numbers. For continuously distributed variables, student's T-test was used. P-Value of 0.05 or less was considered to be significant. For multivariate analysis, the effect of multiple variables on IRI was evaluated with $\mathrm{P}<0.05$ as the threshold for entry into the model.

\section{Results}

A Total of 160 patients of type 2 diabetes with diabetic nephropathy were studied. They were divided into two groups based on intrarenal resistivity index (IRI) by duplex ultrasonography as Group I: Patients with IRI $\leq 0.70(\mathrm{n}=72)$ and Group II: Patients with IRI $>0.70(\mathrm{n}=88)$. The age, sex distribution and symptomatology along with risk factors, complications and renal functions were studied in correlation with IRI.

The mean age in group I was $50.42 \pm 4.89$ and that in group II was $60.34 \pm 7.92(\mathrm{P}=0.000)$. Patients in group II had a significantly higher mean age compared to that of patients of group I The male:female ratio in group I was $1: 1.5$ whereas in group II it was 1:1.2. The comparison of various risk factors between the two groups is shown in Table 1 .

Table 2 shows symptomatology in the two groups.

Mean duration of diabetes mellitus in group I was 4.57 \pm 3.65 years as compared to $11.25 \pm 6.97$ years in group II. Majority of the group I patients namely $62.5 \%$ had less than 5 years duration of diabetes mellitus. Majority of patients of group II had duration of diabetes between 5 to 10 years $(32.95 \%)$ and $11-15$ years $(29.55 \%)$. Thus patients belong- 
Table 4. Urinalysis and Findings on Renal Ultrasound

\begin{tabular}{|c|c|c|c|c|c|}
\hline Investigations & $\begin{array}{l}\text { Group I IRI } \leq 0.70 \\
(\mathrm{n}=72)\end{array}$ & $\begin{array}{l}\text { Group II IRI }>0.70 \\
(n=88)\end{array}$ & Odds ratio & CI & $P$ value \\
\hline Urine $\mathrm{RBC}$ & $03(4.17 \%)$ & $22(25 \%)$ & 7.667 & $2.191-26.829$ & $0.001^{*}$ \\
\hline Sugar & $32(44.44 \%)$ & $30(34.09 \%)$ & 0.647 & $0.341-1.227$ & 0.181 \\
\hline Microalbuminuria & $58(80.55 \%)$ & $15(17.05 \%)$ & 0.05 & $0.022-0.111$ & $<0.001^{*}$ \\
\hline Overt proteinuria & $14(19.44 \%)$ & $73(82.95 \%)$ & 20.162 & $9.007-45.134$ & $<0.001^{*}$ \\
\hline \multicolumn{6}{|l|}{ Renal sonography Kidney size } \\
\hline Right kidney size & $9.7 \times 5.3 \mathrm{~cm}$ & $8.5 \times 4.4 \mathrm{~cm}$ & & & \\
\hline left kidney size & $9.4 \times 5.1 \mathrm{~cm}$ & $8.3 \times 4.5 \mathrm{~cm}$ & & & \\
\hline \multicolumn{6}{|l|}{ Echotexture } \\
\hline 1. Normal & $71(88.88 \%)$ & $69(78.40 \%)$ & 0.051 & $0.007-0.303$ & $<0.001^{*}$ \\
\hline 2. Raised & $01(11.11 \%)$ & $19(21.60 \%)$ & & & \\
\hline \multicolumn{6}{|l|}{ CMD } \\
\hline 1. Normal & $70(97.20 \%)$ & $76(86.36 \%)$ & 0.181 & $0.039-0.837$ & $0.033^{*}$ \\
\hline 2. Altered & $02(2.77 \%)$ & $2(2.27 \%)$ & & & \\
\hline 3. Lost & - & $10(11.36 \%)$ & & & \\
\hline
\end{tabular}

ing to group II had a higher mean duration of diabetes mellitus compared to that of group I.

A total of 46 patients had duration of diabetes less than 5 years with a mean IRI of $0.67 \pm 0.08$ while 67 patients had 5 to 10 years duration of diabetes with a mean IRI of $0.71 \pm 0.08$. There were 29 patients with duration of diabetes between 11 to 15 years with a mean IRI of $0.81 \pm 0.07$. There were 18 patients with duration of diabetes more than 15 years with a mean IRI of $0.89 \pm 0.07$. Thus mean IRI significantly increased with the increase in the duration of diabetes $(\mathrm{P}<0.001)$.

In both the groups, maximum number of patients namely $91.67 \%$ patients in group I and $45.45 \%$ patients in group II were on oral hypoglycaemic agents alone, $38.63 \%$ patients in group II were on insulin and $15.91 \%$ patients were on insulin plus OHA. Patients in group II with ESRD had a lesser requirement for antidiabetics and all of them were on insulin.

Table 3 shows various complications of diabetes in the two groups.

Mean systolic BP in group II was $144.09 \pm 16.79 \mathrm{mmHg}$

Table 5. Kidney Function Test and IRI

\begin{tabular}{llll}
\hline & $\begin{array}{l}\text { Group I } \\
\text { IRI } \leq \mathbf{0 . 7 0}\end{array}$ & $\begin{array}{l}\text { Group II } \\
\text { IRI }>\mathbf{0 . 7 0}\end{array}$ & P value \\
\hline Mean blood urea (mg/dL) & $31.98 \pm 8.99$ & $49.84 \pm 22.65$ & $<0.001^{*}$ \\
Mean Sr. creat (mg/dL) & $0.99 \pm 0.35$ & $2.29 \pm 1.46$ & $<0.001^{*}$ \\
Creatinine clearance (mL/min) & $80.33 \pm 27.01$ & $39.72 \pm 22.37$ & $<0.001^{*}$ \\
Mean blood sugar fasting $(\mathrm{mg} / \mathrm{dL})$ & $101.94 \pm 22.13$ & $98.29 \pm 21.2$ & 0.290 \\
Mean blood sugar post meal $(\mathrm{mg} / \mathrm{dL})$ & $152.85 \pm 31.12$ & $153.86 \pm 30.31$ & 0.836 \\
\hline
\end{tabular}


Table 6. Multiple Linear Regression Analysis of Various Factors Affecting IRI

\begin{tabular}{|c|c|c|c|c|c|}
\hline \multirow{2}{*}{ Model } & \multicolumn{2}{|c|}{ Unstandardized Coefficients } & $\begin{array}{l}\text { Standardized } \\
\text { Coefficients }\end{array}$ & \multirow{2}{*}{$\mathbf{t}$} & \multirow{2}{*}{ Sig. } \\
\hline & B & Std. Error & Beta & & \\
\hline (Constant) & 0.661 & 0.069 & & 9.634 & 0.000 \\
\hline Age & 0.002 & 0.011 & 0.124 & 1.648 & $0.042^{*}$ \\
\hline Smoking & 0.002 & 0.011 & 0.007 & 0.166 & 0.869 \\
\hline Hypertension & 0.023 & 0.011 & 0.100 & 2.045 & $0.043^{*}$ \\
\hline Dyslipidemia & -0.012 & 0.011 & -0.053 & -1.083 & 0.281 \\
\hline Obesity & -0.001 & 0.001 & -0.037 & -0.814 & 0.417 \\
\hline CAD & 0.029 & 0.016 & 0.185 & 1.776 & $0.038^{*}$ \\
\hline Neuropathy & -0.006 & 0.014 & -0.024 & -0.432 & 0.667 \\
\hline Macroalbumin & -0.055 & 0.015 & -0.247 & -3.730 & $0.000^{*}$ \\
\hline Urine $\mathrm{RBC}$ & -0.010 & 0.013 & -0.033 & -0.727 & 0.469 \\
\hline PSV & 0.072 & 0.013 & 0.323 & 5.740 & $0.000^{*}$ \\
\hline Echotexture & 0.023 & 0.019 & 0.082 & 1.246 & 0.215 \\
\hline CMD & 0.048 & 0.033 & 0.114 & 1.439 & 0.152 \\
\hline Retinopathy & -0.045 & 0.013 & -0.132 & -2.565 & $0.048^{*}$ \\
\hline Bld urea & 0.000 & 0.001 & 0.025 & 0.244 & 0.808 \\
\hline Sr.creat & 0.035 & 0.013 & 0.279 & 1.517 & $0.006^{*}$ \\
\hline GFR & 0.040 & 0.070 & -0.092 & -1.066 & $0.045^{*}$ \\
\hline
\end{tabular}

whereas in group I it was $128.47 \pm 13.07 \mathrm{mmHg}$. Mean diastolic BP in group II was $89.31 \pm 9.19 \mathrm{mmHg}$ whereas in group I it was $82.22 \pm 7.59 \mathrm{mmHg}$. Mean systolic and diastolic blood pressure was higher in group II compared to that of group I. There was no significant difference in the mean BMI of both the groups.

Maximum patients $(80.55 \%)$ in group I were in the early stage of nephropathy whereas maximum patients of group II $(69.32 \%)$ were in the established stage of nephropathy. A total of $13.63 \%$ patients were in the ESRD group. This implies that raised IRI is seen more frequently in the established stage of nephropathy and ESRD $(\mathrm{P}=0.000)$. The correlation of findings on urinalysis and renal ultrasound is shown in Table 4.

Table 5 shows renal function tests and blood sugar levels in the two groups.

Table 6 shows multiple linear regression analysis of var- ious factors affecting IRI.

\section{Discussion}

\section{Age and sex distribution}

Mean age of the patients with raised IRI was significantly higher compared to the mean age of the patients with normal IRI. Our findings were similar to those by Platt et al [2] who observed that patients with IRI $>0.70$ were older as compared to those with IRI $\leq 0.70$ (62 years vs 42 years). However Milovanceva-Popovska et al [3] and Nosadini et al [4] did not find any statistically significant difference in the age of patients with raised IRI and those with normal IRI. This could be due to the different cut off value for IRI and also because they had included patients with early nephropathy. 
Based on our observations it can be said that older patients have elevated IRI. It could be because stage of nephropathy advances as age increases and prevalence of associated IRI also increases.

\section{Risk factors and IRI}

There were $36.36 \%$ smokers in group II compared to that of $20.83 \%$ in group I. Our findings were similar to those by Nosadini et al [4] who observed that smoking habit was present in a significant number of patients with IRI $>0.80$ compare to that with IRI $<0.80$. According to Chuahirun et al [5], the proportion of patients affected by low GFR was significantly higher in current smokers. According to Biesenbach et al [6] and Gambaro et al [7] smoking increases the risks of developing microalbuminuria in Type 2 diabetes. Thus Smoking is proved to be a risk factor for diabetic nephropathy. Renal function declines faster in smokers than nonsmokers with type 2 diabetes with nephropathy. Smoking accelerates atherosclerosis and nicotine increases the vascular resistance. It also predisposes to hypertension. Thus the IRI also increases with smoking.

According to our study, $54.55 \%$ patients in group II had hypertension compared to that of $23.61 \%$ in group I which was statistically significant. There are various studies supporting the correlation of hypertension and IRI. According to Ishimura et al [8], there was a significant correlation between blood pressure and IRI. According to Amini et al [9] observed that with presence of hypertension there was a three times higher risk and faster progression of diabetic nephropathy. Thus hypertension has an impact on the IRI especially through the effect of renin angiotensin system on the renal vascular resistance.

As per our study, $56.82 \%$ patients in group II had obesity as risk factor compared to that of $38.89 \%$ in group I. According to Jude et al [10], BMI was significantly higher in patients with nephropathy than those without nephropathy. Similarly Aryal et al [11] observed that increased BMI is associated with faster progression of diabetic nephropathy.

Dyslipidemia was present in $60.23 \%$ in group II compared to that of $30.56 \%$ in group I. Nosadini et al [4] observed that the mean plasma cholesterol was significantly elevated in patients with IRI $>0.80$ compared to those with IRI $<0.80(221 \pm 15$ vs $189 \pm 15)$. According to Aryal et al [11], dyslipidemia was significant in the form of elevated serum cholesterol, LDL and triglycerides in those with nephropathy than those without nephropathy. With the occurrence of alteration in lipid profile in diabetics, there is increased atherogenesis. Also there is ongoing inflammation in the vessel wall which accelerates atherosclerosis.

\section{Symptomatology of diabetic nephropathy and IRI}

In our study more number of patients with increased IRI were symptomatic compared to those with normal $(78.41 \%$ vs $36.11 \%$ ). This is because of the progression of the stage of nephropathy. Our findings were similar to those by Milovanceva-Popovska et al [3] according to whom most of the patients were asymptomatic in the early stage of nephropathy. According to Platt et al [2], the patients were asymptomatic in the early stage of nephropathy characterised only by microalbuminuria whereas those with established nephropathy have some clinical manifestations associated with development of macroalbuminuria. As the IRI increases, and so the stage of nephropathy progresses towards established stage and ESRD, more number of patients become symptomatic for nephropathy and its complications.

\section{Duration of diabetes and IRI}

The mean duration of diabetes mellitus patients with IRI $>$ 0.70 was significantly higher compared to those with normal IRI(11.25 \pm 6.97 vs $4.57 \pm 3.65$ years $)$. Similarly Platt et al [2] observed that patients with IRI more than 0.70 had a mean duration of diabetes of 20 years vs 11 years in those with IRI $<0.70$ which was statistically significant. However Milovanceva-Popovska et al [3] and Nosadini et al [4] did not find any statistically significant difference between the duration of diabetes and IRI. This difference from our study may be due to the lower cut off value for IRI (0.70) and also the difference in the epidemiologic parameters of the study groups.

\section{Complications of diabetes}

Patients with IRI $>0.70$ had coronary artery disease in $19.32 \%$ patients compared to that of $1.39 \%$ with normal IRI. Similarly retinopathy (79.54\% vs $27.78 \%$ ), Neuropathy (40.90\% vs $9.72 \%)$ were observed more in patients with elevated IRI. There was more number of patients with PVD in group II however it was statistically insignificant. According to Nosadini et al [4], a significant number of patients with IRI $>0.80$ had coronary artery disease compared to those with IRI $<0.80(69.69 \%$ vs $12.24 \%)$ and Peripheral vascular disease $(67.79 \%$ vs $19.38 \%)$.

As per the observations of Ishimura et al [8], Diabetic retinopathy was present in a significantly higher number of patients with IRI $>0.80$ compared to those with IRI $<0.80$. Amongst the various stages of diabetic retinopathy, preproliferative and proliferative stage of diabetic retinopathy was more common in patients with IRI $>0.80$.

Thus with the progression of diabetic nephropathy, the other microvascular complications like retinopathy and neuropathy also increase. Also there is increase in the macrovascular complications like coronary artery disease. Basic pathogenesis is diabetes related vasculopathy both micro and macrovascular injury leading to these complications and the rise in intrarenal resistive index. 


\section{Stage of nephropathy and mean IRI}

A total of $80.55 \%$ patients with normal IRI were in the early stage of nephropathy compared to just $19.45 \%$ patients with IRI $>0.70$ whereas $69.32 \%$ with IRI $>0.70$ were in the established stage of nephropathy compared to just $17.05 \%$ with normal IRI. All patients with ESRD had elevated IRI. This implies that raised IRI is seen more frequently in the established stage of nephropathy and ESRD. As the stage of nephropathy progresses from early stage towards established stage and ESRD, the mean IRI also increases.

Platt et al [2] observed that as the stage of nephropathy progresses towards the established stage, IRI significantly increases. Thus, IRI is typically elevated in established nephropathy but is often normal in the early clinical stages of disease.

Ishimura et al [8] studied the Intrarenal hemodynamic abnormalities in diabetic nephropathy. IRI was elevated in diabetic nephropathy, usually at an advanced stage. According to Lee et al [12], intrarenal hemodynamic abnormalities are present in diabetic patients with nephropathy, even in early stage and the intrarenal hemodynamics significantly reflects the stage of nephropathy. This variation may be due to the different cut off valur for IRI.

In advanced DN, sclerotic glomeruli and increased interstitial fibrosis may cause elevated RI values. The RI of interlobar arteries seems to be a dependable marker of intrarenal changes. Activation of the renin-angiotensin system is reported to contribute to inrarenal haemodynamic abnormality in diabetic patients.

\section{Renal function and IRI}

Hematuria was present in a significant number of patients with elevated IRI compared to those with normal IRI. $82.95 \%$ patients with IRI $>0.70$ had overt proteinuria compared to that of only $19.44 \%$ patients with normal IRI. Thus according to our study, proteinuria is significantly associated with raised IRI.

According to Milovanceva-Popovska et al [3], IRI is significantly affected by the proteinuria. Similarly Nosadini et al [4] observed that $24 \%$ of the patients with IRI $>0.80$ had overt proteinuria compared to that of just $5 \%$ patients with IRI $<0.80$ which was statistically significant. According to Jude et al [10] proteinuria was significantly higher in the established stage of nephropathy group.

Thus Microalbuminuria is not merely a predictor of diabetic nephropathy but also constitutes evidence of renal damage. IRI $\geq 0.70$ can predict the outcome of renal function in type 2 diabetic patients with microalbuminuria. Presence of microalbuminuria has been identified as a possible predictor of subsequent overt diabetic nephropathy in patients without dipstick- sensitive proteinuria. With the appearance of overt proteinuria, IRI also increases significantly.

\section{Kidney function tests and IRI}

Patients with an IRI $>0.70$ had a significantly high blood urea $(49.84 \pm 22.65$ vs $31.98 \pm 8.99 \mathrm{mg} / \mathrm{dL})$ and serum creatinine $(2.29 \pm 1.46$ vs $0.99 \pm 0.35 \mathrm{mg} / \mathrm{dL})$ compared to those with normal IRI. The mean creatinine clearance was significantly reduced with IRI $>0.70$ compared to those with normal IRI $(39.72 \pm 22.37$ vs $80.33 \pm 27.01 \mathrm{~mL} / \mathrm{min})$. Our findings were similar to those by Milovanceva-Popovs$\mathrm{ka}$ et al [3] who observed that the mean creatinine clearance in patients with IRI $>0.70$ was $47.4 \pm 4.9$ compared to that of $51.4 \pm 7.6$ with IRI $<0.70$ which was statistically significant. Similarly Platt et al [2] observed that patients with IRI more than 0.70 had a mean serum creatinine of $3.2 \mathrm{mg} / \mathrm{dL}$ compared to that of $1.1 \mathrm{mg} / \mathrm{dL}$ in patients with IRI $<0.70$. Similarly according to Nosadini et al [4], the mean creatinine clearance in patients with IRI $<0.80$ was $91 \pm 7 \mathrm{~mL} / \mathrm{min}$ whereas in patients with IRI $>0.80$ it was $70 \pm 8 \mathrm{~mL} / \mathrm{min}$. IRI might be useful to identify the cohort of microalbuminuric patients with more severe renal lesions and those prone to develop a rapid decay of GFR without performing routinely the invasive procedure of renal biopsy and IRI $>0.80$ had a strong correlation with the GFR and creatinine clearance. Thus with the decline in renal function reflected by the serum reatinine and creatinine clearance, the intrarenal hemodynamics are also altered reflected by the rise in IRI.

\section{Renal sonography and IRI}

The mean kidney size in patients with normal IRI was not significantly different from those with elevated IRI $(9.7 \times 5.3$ and $9.4 \times 5.1 \mathrm{~cm}$ vs $8.5 \times 4.4$ and $8.3 \times 4.5 \mathrm{~cm}$ ). CMD was mostly affected only in ESRD. According to Majdan et al [13] who studied patients of diabetic nephropathy with and without chronic renal failure found that most of Type $2 \mathrm{DM}$ patients with CRF had small kidneys which mean they had ischemic, hypertonic or inflammatory nephropathy accompanying Type 2 Diabetes.

A total of $70.45 \%$ patients with elevated IRI had elevated Peak systolic velocity $(>150 \mathrm{~cm} / \mathrm{s})$ compared to that of just $6 \%$ patients with a normal IRI. Studies carried out by Platt et al [2], Nosadini et al [4], Ishimura et al [8] showed a positive correlation between PSV, Stage of nephropathy and IRI. On multivariate analysis, it was observed that factors which independently affected IRI were Age, Hypertension, Complications - Coronary artery disease and retinopathy, Macroalbuminuria, Decreased creatinine clearance.

Thus we conclude that Intrarenal resistivity index as assessed by duplex ultrasonography is a non-invasive parameter that can be correlated with the clinical profile and biochemical parameters of renal dysfunction in patients of type II Diabetes mellitus with diabetic nephropathy. It correlates significantly with worsening renal function. 


\section{Financial Disclosures}

Nil.

\section{Declaration}

Name and full location of department and institution where work was performed: Department of Medicine, Government Medical College, Nagpur, Maharashtra, India.

\section{Grant Support}

Nil.

\section{References}

1. Ramachandran A, Snehalatha C, Satyavani K, Latha E, Sasikala R, Vijay V. Prevalence of vascular complications and their risk factors in type 2 diabetes. J Assoc Physicians India. 1999;47(12):1152-1156.

2. Platt JF, Rubin JM, Ellis JH. Diabetic nephropathy: evaluation with renal duplex Doppler US. Radiology. 1994;190(2):343-346.

3. Milovanceva-Popovska M, Dzikova S. Progression of diabetic nephropathy: value of intrarenal resistive index (RI). Prilozi. 2007;28(1):69-79.

4. Nosadini R, Velussi M, Brocco E, Abaterusso C, Carraro A, Piarulli F, Morgia G, et al. Increased renal arterial resistance predicts the course of renal function in type 2 diabetes with microalbuminuria. Diabetes. 2006;55(1):234-239.

5. Chuahirun T, Wesson DE. Cigarette smoking predicts faster progression of type 2 established diabetic nephropathy despite ACE inhibition. Am J Kidney Dis. 2002;39(2):376-382.

6. Biesenbach G, Grafinger P, Janko O, Zazgornik J. Influence of cigarette-smoking on the progression of clinical diabetic nephropathy in type 2 diabetic patients. Clin Nephrol. 1997;48(3):146-150.

7. Gambaro G, Bax G, Fusaro M, Normanno M, Manani SM, Zanella M, Dangelo A, et al. Cigarette smoking is a risk factor for nephropathy and its progression in type 2 diabetes mellitus. Diabetes Nutr Metab. 2001;14(6):337-342.

8. Ishimura E, Nishizawa Y, Kawagishi T, Okuno Y, Kogawa K, Fukumoto S, Maekawa K, et al. Intrarenal hemodynamic abnormalities in diabetic nephropathy measured by duplex Doppler sonography. Kidney Int. 1997;51(6):1920-1927.

9. Amini M, Safaei H, Aminorroaya A. The incidence of microalbuminuria and its associated risk factors in type 2 diabetic patients in isfahan, iran. Rev Diabet Stud. 2007;4(4):242-248.

10. Jude EB, Anderson SG, Cruickshank JK, Srivatsa A, Tentolouris N, Chandrasekaran R, Gokal R, et al. Natural history and prognostic factors of diabetic nephropathy in type 2 diabetes. QJM. 2002;95(6):371-377.

11. Aryal M, Jha B. Assessment of proteinuria as a marker of nephropathy in type 2 diabetes mellitus. Nepal Med Coll J. 2006;8(4):250-253.

12. Lee JH, Jung YD, Shon HS, Ahn KS, Chung DS. The Difference of Intrarenal Hemodynamics in Type 2 Diabetic Nephropathy. J Korean Diabetes Assoc 1999 Dec 23(6):822-830. Korean.

13. Majdan M, Kurowska M, Orlowska-Kowalik G, Drop A. [Ultrasonographic evaluation of kidneys in type-2 diabetes patients without overt nephropathy and with chronic renal failure]. Wiad Lek. 2005;58(1-2):25-28. 